\title{
ON A THEOREM OF FELL
}

\begin{abstract}
ROBERT C. BUSBY
ABSTRACT. Fell has proved that the process of inducing representations of a locally compact group from representations of closed subgroups is a continuous process if topologies are defined on the spaces of representations in the right way. As a corollary he shows that inducing preserves weak containment. This paper generalizes Fell's results to twisted group algebras. These algebras generalize the idea of the group algebra of a group extension, and the concept of induced representation extends in a natural way. We show that Fell's results will hold if the "cocycle pair" defining the twisting of the algebra is sufficiently continuous.
\end{abstract}

Preliminaries. In [4] and [5], Fell discussed weak containment properties of induced representations of groups, and in particular he discussed the continuity of the inducing construction with respect to two topologies related to weak containment. Group algebras of group extensions are special cases of twisted group algebras as defined in [1], and in [1] the concept of induced representation was extended to these algebras. It is desirable to extend some of Fell's results, especially his main continuity theorem [4, Theorem 4.1], to the more general case. Such results are needed for the example in [2] in the study of the compactness of induced representations. We prove this extension in the present note.

Throughout this paper, $G$ will be a fixed second countable, locally compact group with left Haar measure $\mu$ and corresponding modular function $\Delta$, and $A$ will be a fixed separable Banach ${ }^{*}$-algebra with a bounded, two-sided approximate identity. Let $M(A)$ and $\mathfrak{u}(A)$ denote respectively the double centralizer algebra of $A$ and the group of unitaries in $M(A)$, each of these having the strict topology (see [1] for definitions), and let $\operatorname{Aut}_{1}(A)$ be the group of isometric *-automorphisms of $A$ with the pointwise convergence topology. A twisting pair for $(G, A)$ is a pair $(T, \alpha)$ where $\alpha: G \times G \rightarrow \mathcal{U}(A)$ and $T: G \rightarrow$ Aut $_{1}(A)$ are Borel functions such that:

(1) $T(x) \alpha(y, z) \alpha(x, y z)=\alpha(x, y) \alpha(x y, z)$ (the common value of these expressions will from now on be denoted $\beta(x, y, z))$.

(2) $(T(x) T(y) a) \alpha(x, y)=\alpha(x, y)(T(x y) a) ; \alpha(x, e)=\alpha(e, y)=I$.

Received by the editors November 18, 1970 .

AMS 1969 subject classifications. Primary 4650, 4680; Secondary 2260.

Key words and phrases. Twisted group algebra, induced representation, inner hullkernel topology, quotient topology, weak containment. 
(3) There is a neighborhood $N$ of $e$ on $G$ such that the restrictions of $T$ and $\alpha$ to $N$ and $N \times N$ respectively are continuous.

(4) For each $x$ in $G$ there is a neighborhood $N_{x}$ of $e$ in $G$ such that $\beta\left(x^{-1}, y, x\right)$ is continuous in $N_{x}$ as a function of $y$.

In all the above, $x, y, z$ are in $G, a$ is in $A, e$ is the identity in $G$, and $I$ is the identity in $M(A)$.

In [1], twisting pair was defined without the continuity conditions (3) and (4) above, but it does not seem possible to generalize Fell's theorem without some continuity condition. Our assumptions in this direction are fairly weak, and in the special case of a group extension they are implied by the existence of a cross-section continuous in a neighborhood of the identity (this exists for instance when the groups are Lie groups).

The Banach space $L^{1}(G, A)$ of Bochner integrable, $A$-valued functions becomes a Banach ${ }^{*}$-algebra if for $f, h$ in $L^{1}(G, A)$ and $x, y$ in $G$ we define:

(5) $(f \circ h)(x)=\int_{G} f(y)\left(T(y) g\left(y^{-1} x\right)\right) \alpha\left(y, y^{-1} x\right) d \mu(y)$ and

(6) $f^{*}(x)=\alpha\left(x, x^{-1}\right)^{*}\left(T(x) f\left(x^{-1}\right)^{*}\right) \Delta(x)^{1 / 2}$.

The resulting algebra, denoted $L^{1}(G, A ; T, \alpha)$, is called a twisted group algebra. If $p: G \rightarrow u(A)$ is Borel, we can define a new twisting pair $\left(T_{p}, \alpha_{p}\right)$ by letting $T_{p}(x) a=p(x)(T(x) a) p(x)^{*}$ and $\alpha_{p}(x, y)$ $=p(x)(T(x) p(y)) \alpha(x, y) p(x y)^{*}$. Then the map $\Phi_{p}$ from $L^{1}(G, A ; T, \alpha)$ to $L^{1}\left(G, A ; T_{p}, \alpha_{p}\right)$ defined by $\Phi_{p}(f)(x)=f(x) p(x)^{*}$ is an isometric *-isomorphism. We say that two pairs $(T, \alpha)$ and $\left(T^{\prime}, \alpha^{\prime}\right)$ are equivalent if there is a mapping $p$ as above such that $\left(T^{\prime}, \alpha^{\prime}\right)=\left(T_{p}, \alpha_{p}\right)$.

Finally, let $\pi$ be a representation (i.e. nondegenerate ${ }^{*}$-representation) of $A$ on a Hilbert space $H$, let $\mathcal{H C}=L^{2}(G, H)$, the space of Bochner square-integrable (resp. $\mu$ ) functions, and define, for each $x$ in $G$ and $a$ in $A$, operators $U^{x}(x)$ and $\tilde{\pi}(a)$ on $\mathcal{H}$ by:

(7) $\left(U^{x}(x) h\right)(y)=\pi(\alpha(y, x)) h(y x) \Delta(x)^{1 / 2}$,

(8) $(\tilde{\pi}(a) h)(x)=\pi(T(x) a) h(x)$.

We then define the representation $\Pi$ of $L^{1}(G, A ; T, \alpha)$ induced from $\pi$ by letting $\Pi(f)=\int_{G} \tilde{\pi}(f(x)) U^{\pi}(x) d \mu(x)$. In the future we will sometimes denote the representation induced from $\pi$ by $I(\pi)$. If $\left(T_{p}, \alpha_{p}\right)$ is a pair constructed from $(T, \alpha)$ as above, then $\pi$ also induces a representation $I_{p}(\pi)$ of $L^{1}\left(G, A ; T_{p}, \alpha_{p}\right)$. It is shown in [3] that $I_{p}(\pi) \circ \Phi_{p}$ is unitarily equivalent with $I(\pi)$ and that this equivalence is produced by the unitary $\Psi_{p}$, where if $h \in \mathcal{H}, \Psi_{p}(h)(x)=\pi(p(x)) h(x)$. Notice that both $\Phi_{p}$ and $\Psi_{p}$ leave the domains of definition of the functions on which they operate pointwise fixed.

2. The main result. We retain in this section, all definitions, notations, and conventions of $\$ 1$. 
LEMMA 1. There is a neighborhood base $B$ for the identity $e$ of $G$ consisting of sets $V$ such that:

(a) $V$ is open, relatively compact, and symmetric.

(b) If $n$ is a positive integer and the symbol bd denotes boundary, then $\mu\left(\mathrm{bd}\left(V^{n}\right)\right)=0$.

Proof. Pick a translation invariant metric $d$ on $G$ (see [6]), and for all real numbers $1 \geqq r>0$ let $U_{r}$ (resp. $C_{r}$ ) be the open (resp. closed) sphere of radius $r$ about $e$ (note that we need not have $\bar{U}_{r}=C_{r}$ ). We may also choose $d$ so that $C_{1}$ is compact. It is trivial that if $n$ is as above, $\left(U_{r}\right)^{n}=U_{s<r}\left(U_{s}\right)^{n}$ and $\left(C_{r}\right)^{n}=\bigcap_{8>r}\left(U_{s}\right)^{n}$. Define a real valued function of the positive variable $r$ by letting $f_{n}(r)=\mu\left(U_{r}^{n}\right)$, and note that for each $n, f_{n}$ is a monotone function and so is continuous except for a countable set. If $f_{n}$ is continuous at a point $r$, the set theoretic inequalities written above yield the fact that $S_{r} \equiv\left(C_{r}\right)^{n}-\left(U_{r}\right)^{n}$ (set theoretic difference) has Haar measure zero.

Since $\left(U_{r}\right)^{n}$ is open and $\left(C_{r}\right)^{n}$ is closed (since it is compact), the boundary of $\left(U_{r}\right)^{n}$ must be contained in $S_{r}$ and so has measure zero. Define $B$ to be the set of all open spheres having as radius a number where each $f_{n}$ is continuous. Even on the real line there are open sets which have boundaries that are compact and have positive measure. The above lemma lets us ignore them.

The next lemma is technical and a little awkward, but it is the main tool for this paper and promises to be helpful in future technical constructions for twisted group algebras. It squeezes enough local continuity out of $\alpha$ (given our rather weak assumptions) to enable us to use some of the usual approximation tricks of analysis.

LEMMA 2. Let $s \in G$. There exist: (1) a neighborhood $U$ of $s$ and a compact set $A^{\prime}$ of measure zero (depending on $s$ ), (2) for each $t$ in the complement of $A^{\prime}$, a neighborhood $V_{t}$ of $t$ and a Borel function $p_{t}$ from $G$ to $\mathfrak{u}(A)$ (both depending on s and $t$ ) such that:

(a) $\alpha_{t}(x, y)$ is continuous for $x$ in $V_{t}$ and $y$ in $U$, and

(b) $T_{t}(x)$ is continuous for $x$ in $U \cup V_{t}$.

Here $\left(T_{t}, \alpha_{t}\right)$ is the pair constructed from $(T, \alpha)$ through $p_{t}$.

(c) $p_{t_{1}}(x)=p_{t_{2}}(x)$ for all $x$ in $U, t_{1}$ and $t_{2}$ in the complement of $A^{\prime}$.

Proof. Suppose first that $s$ is not the identity. Choose $W$ from $B$ (see Lemma 1) such that $W^{2} \cup s^{-1} W^{2} s \cup s W^{2} s^{-1} \subset N \cap N_{s}$, and $s \notin W^{5}$. Let $U=s W$ and $A^{\prime}=\mathrm{bd}(U) \cup \mathrm{bd}\left(U U^{-1}\right)$ (which has measure zero by hypothesis), and suppose that $t \in A^{\prime}$. We consider cases.

Case 1. $t \notin U \cup U U^{-1}$. Our hypotheses imply that we can choose a symmetric neighborhood $V$ of $e$ such that 
(1) $V^{2} \cap U=\varnothing$,

(2) $t V U \cap U=\varnothing$,

(3) $t V \cap U=\varnothing$,

(4) $s^{-1} V s \subset N$,

(5) $V \subset N_{8}$.

Each of these things is possible either because of our assumptions on $s$ or the fact that $t$ is not in $U, U U^{-1}$, or the boundaries of these sets. (1), (2), and (3) tell us that the three sets $t V, U$, and $t V U$ are mutually disjoint. We then define $p_{t}$ as follows: if $x=t v$ is in $t V$, let $p_{t}(x)$ $=\alpha(t, v)$; if $x=s w$ is in $U$, let $p_{t}(x)=\alpha(s, w)$; if $x=t s w^{\prime}$ (with $w^{\prime}$ $\in s^{-1} V s W$ ) is in $t V U$ (and every point in $t V U$ can be written this way uniquely) then we let $p_{t}(x)=\alpha\left(t s, w^{\prime}\right)$. At all other points, $p_{t}$ is the identity in $M(A)$. Now suppose that $x \in t V$, and $y \in U$, say $x=t v$ and $y=s w$. We compute $\alpha_{t}(x, y)$ :

$$
\begin{aligned}
\alpha_{t}(x, y) & =\left(\text { by definition) } p_{t}(x)\left(T(x) p_{t}(y)\right) \alpha(x, y) p_{t}(x y)^{*}\right. \\
& =\alpha(t, v)(T(t v) \alpha(s, w)) \alpha(t v, s w) \alpha\left(t s, w^{\prime}\right)^{*} \quad\left(\text { where } w^{\prime}=s^{-1} v s w\right) \\
& =(T(t) T(v) \alpha(s, w)) \alpha(t, v) \alpha(t v, s w) \alpha\left(t s, w^{\prime}\right)^{*} \\
& =(T(t) T(v) \alpha(s, w))(T(t) \alpha(v, s w)) \alpha(t, v s w) \alpha\left(t s, w^{\prime}\right)^{*} \\
& =T(t)(\alpha(v, s) \alpha(v s, w)) \alpha(t, v s w) \alpha\left(t s, w^{\prime}\right)^{*} \\
& =T(t)(\alpha(v, s) \alpha(v s, w))\left(T(t) \alpha\left(s, s^{-1} v s w\right)^{*}\right) \alpha(t, s) \\
& =T(t)\left(\alpha(v, s) \alpha\left(s, s^{-1}\right)^{*}\left(\alpha\left(s, s^{-1}\right) \alpha\left(s, s^{-1} v s\right)^{*}\right)\right. \\
& \left.\quad \cdot\left(\alpha\left(s, s^{-1} v s\right) \alpha(v s, w) \alpha\left(s, s^{-1} v s w\right)^{*}\right)\right) \alpha(t, s) \\
& =T(t)\left(\alpha(v, s)\left(T(s) \alpha\left(s^{-1}, s\right)^{*}\right)\left(T(s) \alpha\left(s^{-1}, v s\right)\right)\left(T(s) \alpha\left(s^{-1} v s, w\right)\right)\right) \alpha(t, s) \\
& =T(t) T(s)\left(T(s)^{-1} \alpha(v, s) \alpha\left(s^{-1}, s\right)^{*} \alpha\left(s^{-1}, v s\right) \alpha\left(s^{-1} v s, w\right)\right) \alpha(t, s) \\
& =T(t) T(s)\left(\alpha\left(s^{-1}, s\right)^{*}\left(\left(T\left(s^{-1}\right) \alpha(v, s)\right) \alpha\left(s^{-1}, v s\right)\right) \alpha\left(s^{-1} v s, w\right)\right) \alpha(t, s) \\
& =T(t) T(s)\left(\alpha\left(s^{-1}, s\right)^{*} \beta\left(s^{-1}, v, s\right) \alpha\left(s^{-1} v s, w\right)\right) \alpha(t, s) .
\end{aligned}
$$

Since $\alpha$ is continuous on $s^{-1} V s \times W$ and $\beta\left(s^{-1}, v, s\right)$ is continuous for $v$ in $V$, and since $s$ and $t$ are constant, $\alpha_{t}(x, y)$ is continuous in $V_{t} \times U$, where $V_{t}=t V$. A simple computation also shows that if $x$ is in $t V$, $T_{t}(x)=T(t) T(v)$, and similarly in $U$; thus $T_{t}$ is continuous in $V_{t} \cup U$.

Having illustrated the point we will spare the reader most of the details for the other cases. In case two we have $t \in U U^{-1}$, and we note that $U U^{-1}, U$, and $s W^{3}$ are mutually disjoint (since $\left.s \notin W^{5}\right)$. If $x$ is in $U U^{-1}$ and $y$ is in $U, x y$ is in $s W^{3}$. We let $p_{t}(y)=\alpha(s, w)$ if $y$ $=s w$ is in $U$, and $p_{t}(z)=\alpha\left(s, w^{\prime}\right)$ if $z=s w^{\prime}$ is in $s W^{3}$. Otherwise $p_{t}$ is the identity. Again a long computation shows that if $x$ is in $U U^{-1}$ and $y$ is in $U$, then $\alpha_{t}(x, y)=T(s)\left(\alpha\left(s^{-1}, s\right)^{*} \beta\left(s^{-1}, x, s\right) \alpha\left(s^{-1} x s, w\right)\right)$ and since 
$\alpha$ is continuous on $W^{2} \times W$ and $s W^{2} s^{-1} \subset N_{s}, \alpha_{t}$ is continuous on $V_{t}$ $\times U$ where $V_{t}=U U^{-1} . T_{t}$ is continuous on $V_{t} \cup U$ as before.

In the final case, $t \in U$. Our assumptions show that $U^{2} \cap U=\varnothing$. If $x=s w$ is in $U$, let $p_{t}(x)=\alpha(s, w)$, and if $x=s^{2} w^{\prime}$ is in $U^{2}\left(w^{\prime} \in s^{-1} W s W\right)$ let $p_{t}(x)=\alpha\left(s^{2}, w^{\prime}\right)$. If $x$ and $y$ are in $U, x=s w_{1}, y=s w_{2}, \alpha_{t}(x, y)$ $=\alpha(s, s) T\left(s^{2}\right)\left(\alpha\left(s^{-1}, s\right) * \beta\left(s^{-1}, w_{1}, s\right) \alpha\left(s^{-1} w_{1} s, w_{2}\right)\right)$, and again our assumptions show that $\alpha_{t}$ is continuous on $V_{t} \times U$ where $V_{t}=U$, and $T_{t}$ is continuous on $V_{t} \cup U$. In all three cases, condition (c) clearly holds by construction. This completes the proof if $s \neq e$. If $s=e$, different but analogous (and somewhat easier) computations yield the same conclusions. We will leave the details of this case to the reader.

Now suppose that for each point $x$ in $G$, with the exception of a set $A^{\prime}$ of measure zero, we are given an open neighborhood $U_{x}$ of $x$ and a set $S_{x}$ of functions with supports in $U_{x}$ such that $S_{x}$ is dense in $L^{p}\left(U_{x}, B, \mu_{x}\right)$, where $B$ is a Banach space and $\mu_{x}$ is the restriction of Haar measure $\mu$ to $U_{x} . L^{p}\left(U_{x}, B, \mu_{x}\right)$ has the usual meaning.

LEMмa 3. The set $S$ of finite linear combinations of functions in the sets $S_{x}$ form a dense set in $L^{p}(G, B, \mu)$ (under the obvious inclusion of the spaces $L^{p}\left(U_{x}, B, \mu_{x}\right)$ in $\left.L^{p}(G, B, \mu)\right)$.

Proof. By the classical method of Vitali, we can find a set $M$ with complement of Haar measure zero such that $M$ is covered by a disjoint union $S$ of open sets, each of which is a subset of some $U_{x}(M$ may be taken to include the set $A^{\prime}$ ). It is clear that any function $f$ in $L^{p}(G, B, \mu)$ can be written as a converging series $\sum_{n=1}^{\infty} f_{n}$ where each $f_{n}$ is the restriction of $f$ to some set in the covering. Each $f_{n}$ can then be approximated by functions in some $S_{x}$, and the lemma follows. We remark that linear combinations of functions $f \otimes b, b$ in $B$ and $f$ positive real valued continuous, where $(f \otimes b)(x)=f(x) b$, are dense in $L^{p}(G, B, \mu)$ (and for any $x$ and functions $f$ with support in $U_{x}$ these combinations are dense in $\left.L^{p}\left(U_{x}, B, \mu_{x}\right)\right)$. This fact can easily be seen to be true from material in the integration section of Dunford and Schwartz, vol. I, so the proof is complete.

Now suppose that $\left(\pi_{j}\right)_{j \in J}$ is a net of representations of $A$ on a Hilbert space $H$ and that there is a representation $\pi$ of $A$ on $H$ such that $\left\|\pi_{j}(a) \xi-\pi(a) \xi\right\| \rightarrow 0$ as $j \rightarrow \infty$ for each $a$ in $A$ and $\xi$ in $H$.

LeMma 4. Let $U$ and $V$ be open, relatively compact sets in $G$ such that $\alpha$ is continuous on $U \times V$, and $T$ is continuous on $U \cup V$, where $(T, \alpha)$ is a twisting pair for $(G, A)$. Then if $F$ is in $L^{1}(U, A)$ and $\Gamma$ is in $L^{2}(V, H)$ (both with respect to Haar measure) we have $\left(I\left(\pi_{j}\right)(F) \Gamma \mid \Gamma\right) \rightarrow$ 
$(I(\pi)(F) \Gamma \mid \Gamma)$ as $j \rightarrow \infty$ ( $I$ is the inducing operation and in any Hilbert space $(\mid)$ is inner product).

Proof. Let $F=a \otimes f$ where $f$ is continuous and real valued with support in $U$ and $a$ is in $A$. Also let $\Gamma=\xi \otimes \gamma$ where $\gamma$ is continuous and real valued with support in $V$ and $\xi \in H$. Then we have:

$$
\left(\left(I\left(\pi_{j}\right)(F)\right) \Gamma\right)(x)=\int_{G} \pi_{j}(T(x) a) \pi_{j}(\alpha(x, y)) \xi f(y) \gamma(x y) \Delta(y)^{1 / 2} d \mu(y)
$$

and similarly for $((I(\pi)(F)) \Gamma)(x)$. Thus we have

$$
\begin{aligned}
&\left(\left(I\left(\pi_{j}\right)(F)-I(\pi)(F)\right) \Gamma \mid \Gamma\right) \\
& \quad=\int_{G} \int_{G}\left(\left(\pi_{j}(T(x) a \alpha(x, y))-\right.\right.\pi(T(x) a \alpha(x, y))) \xi \mid \xi) \\
& \cdot f(y) \gamma(x y) \gamma(x) \Delta(y)^{1 / 2} d \mu(y) d \mu(x) .
\end{aligned}
$$

By choice of $f$ and $\gamma$, we see that the integrand vanishes unless $y \in U$ and $x \in V$ and in these sets the functions $T$ and $\alpha$ are continuous. Partition $U \times V$ into disjoint Borel sets $M_{q}$ such that the variation of the function $T(x) a \alpha(x, y)$ is less than $\epsilon / 3$ in each such set. Let $a_{q}=T\left(x_{q}\right) a \alpha\left(x_{q}, y_{q}\right)$ for some fixed point $\left(x_{q}, y_{q}\right)$ in each $M_{q}$, and choose $j_{0}$ so large that, if $j>j_{0},\left\|\pi_{j}\left(a_{q}\right)-\pi\left(a_{q}\right)\right\|<\epsilon / 3$ for all $q$. It follows by the usual approximation argument that, if $j>j_{0}$, $x \in V, y \in U$, then

$$
\left\|\pi_{j}(T(x) a \alpha(x, y))-\pi(T(x) a \alpha(x, y))\right\|<\epsilon .
$$

Then the absolute value of the quantity $\left(^{*}\right)$ above does not exceed $\epsilon\|\xi\|_{2}^{2}\|f\|_{\infty}\|\gamma\|_{\infty}^{2} M$ where $M$ is the maximum of the function $\Delta$ on the (compact) closure of $U$. Since $\epsilon$ can be chosen arbitrarily, we see that $\left(I\left(\pi_{j}\right)(F) \Gamma \mid \Gamma\right) \rightarrow(I(\pi)(F) \Gamma \mid \Gamma)$ as $j \rightarrow \infty$. We have remarked previously that linear combinations of functions such as $F$ and $\Gamma$ given above are dense in $L^{1}(U, A)$ and $L^{2}(V, H)$ respectively, and so the linearity and continuity of the integration involved shows that the above conclusions hold for any $F$ in $L^{1}(U, A)$ and $\Gamma$ in $L^{2}(V, H)$.

LEMMA 5. If $\left(\pi_{j}\right)_{j \in J}$ and $\pi$ are as above, then for any $F$ in $L^{1}(G, A ; T, \alpha)$ and $\Gamma$ in $L^{2}(G, H)$ we have $\left(I\left(\pi_{j}\right)(F) \Gamma \mid \Gamma\right) \rightarrow(I(\pi)(F) \Gamma \mid \Gamma)$ as $j \rightarrow \infty$.

Proof. Fix $s$ in $G$ and let $A^{\prime}, U, t \notin A^{\prime}, V_{t}$, and $\left(\alpha_{t}, T_{t}\right)$ be as in Lemma 2. Since $\left(\alpha_{t}, T_{t}\right)$ satisfies the conditions of Lemma 4 on $U \times V_{t}$, we see that

$$
\left(I_{t}\left(\pi_{j}\right)(F) \Gamma \mid \Gamma\right) \rightarrow\left(I_{t}(\pi)(F) \Gamma \mid \Gamma\right) \quad \text { as } j \rightarrow \infty \text { for all } F \text { in } L^{1}(U, A)
$$


and $\Gamma$ in $L^{2}\left(V_{t}, H\right)$ (where $I_{t}$ is the operation of inducing up to $\left.L^{1}\left(G, A ; T_{t}, \alpha_{t}\right)\right)$. Now the isometry $\Phi_{t}$ of $L^{1}(G, A ; T, \alpha)$ to $L^{1}\left(G, A ; T_{t}, \alpha_{t}\right)$ and the unitary $\Psi_{t}$ which, for any representation $\pi$ of $A$, induces an equivalence between $I(\pi)$ and $I_{t}(\pi) \circ \Phi_{t}$, both preserve the supports of the functions on which they operate (see the discussion at the start of this paper). It follows from this that if $F$ is in $L^{1}(U, A)$ and $\Gamma$ is in $L^{2}\left(V_{t}, H\right)$ then $\lim _{j \rightarrow \infty}\left(I\left(\pi_{j}\right)(F) \Gamma \mid \Gamma\right)$ $=(I(\pi)(F) \Gamma \mid \Gamma)$. Lemma 3 applied to functions $\Gamma$ in $L^{2}(G, H)$ which have support in the set $V_{t}$ for some $t$ not in $A^{\prime}$ tells us that $\lim _{j \rightarrow \infty}\left(I\left(\pi_{j}\right)(F) \Gamma \mid \Gamma\right)=(I(\pi)(F) \Gamma \mid \Gamma)$ for every $\Gamma$ in $L^{2}(G, H)$ and $F$ in $L^{1}(U, A)$. We finally let $s$ and $U$ vary and apply Lemma 3 to functions in $L^{1}(G, A)$ with supports in the various sets $U$. This completes the proof of the lemma.

We refer the reader to [4] for the definitions of inner hull-kernel and quotient topologies on the family of representations of an algebra (or group). Now that we have Lemma 5, we can prove our main theorem just as Fell proved Theorem 4.1 of [4]. We refer, the reader to Fell's proof and merely sketch the results in our context.

TheOREM 1. The map I from representations of $A$ to those of $L^{1}(G, A ; T, \alpha)$ is continuous when each of these sets is given the inner hull-kernel topology.

Proof. Suppose that $S$ is a set of representations of $A$ and $\pi$ is in the inner hull-kernel closure of $S$. As Fell shows, we may assume that $\pi$ and the representations in $S$ are all on the same separable Hilbert space $H$, and that there is a net $\pi_{j}$ in $S$ which converges to $\pi$ in the quotient topology, that is for $a$ in $A$ and $\xi$ in $H,\left\|\pi_{j}(a) \xi-\pi(a) \xi\right\| \rightarrow 0$ as $j \rightarrow \infty$. Lemma 5 then tells us that $\left(I\left(\pi_{j}\right)(F) \Gamma \mid \Gamma\right) \rightarrow(I(\pi)(F) \Gamma \mid \Gamma)$ as $j \rightarrow \infty$ for $F$ in $L^{1}(G, A ; T, \alpha)$ and $\Gamma$ in $L^{2}(G, H)$. Reasoning with algebras as Fell does with groups, we conclude that if $\phi_{1}, \cdots, \phi_{n}$ are positive functionals on $L^{1}(G, A ; T, \alpha)$ associated with the representation $I(\pi)$, then there are positive functionals $\psi_{1}, \cdots, \psi_{n}$ associated with $I(S)$ such that $\left|\phi_{i}(F)-\psi_{i}(F)\right|<\epsilon, i=1, \cdots, n$, for any previously chosen $\epsilon$. Thus $I(\pi)$ is in the inner hull-kernel closure of $I(S)$, and we are done. The following corollary follows as in [4].

COROLLARY 1. If $\pi$ is weakly contained in a family $S$ (all are representations of $A$ ), then $I(\pi)$ is weakly contained in the family $I(S)$.

\section{REFERENCES}

1. R. C. Busby and H. A. Smith, Representations of twisted group algebras, Trans. Amer. Math. Soc. 149 (1970), 503-537. 
2. R. C. Busby and Irwin Schochetman, Compact induced representations, Canad. J. Math. (submitted).

3. R. C. Busby, I. Schochetman and H. A. Smith, Integral operators and the compactness of induced representations, Trans. Amer. Math. Soc. (submitted).

4. J. M. G. Fell, Weak containment and induced representations of groups, Canad. J. Math. 14 (1962), 237-268. MR 27 \#242.

5. - Weak containment and induced representations of groups. II, Trans. Amer. Math. Soc. 110 (1964), 424-447. MR 28 \#3114.

6. D. Montgomery and L. Zippin, Topological transformation groups, Interscience, New York, 1955. MR 17, 383.

Drexel University, Philadelphia, Pennsylvania 19104 